\title{
SOME PROPERTIES OF PSEUDO-CONFORMAL IMAGES OF REINHARDT CIRCULAR DOMAINS
}

\author{
STEFAN BERGMAN AND KYONG T. HAHN ${ }^{1}$
}

0. Introduction. A one-to-one mapping of a domain $D$ of $C^{2}$ by a pair of analytic functions, say

$$
T=\left[z_{k}^{*}=z_{k}^{*}\left(z_{1}, z_{2}\right), k=1,2\right],
$$

onto another domain $D^{*}$ is called a PCT (pseudo-conformal transformation). In contrast to the case of simply connected domains in the complex plane $C$, simply connected domains $D$ in $C^{2}$ are as a rule not homogeneous. It is of interest in the theory of PCT's to determine and investigate the "interior distinguished sets"; i.e., sets possessing certain properties which in a PCT go over into sets having the same properties. As indicated in the previous papers [B.3], [B.7], [B.8], [B.9], [B.11], [B.12], the theory of the kernel function enables us to determine certain (absolute) invariants $J_{D}{ }^{(\nu)}\left(z_{1}, z_{2} ; \bar{z}_{1}, \bar{z}_{2}\right) \equiv J_{D}{ }^{(\nu)}(z, \bar{z})$, $z=\left(z_{1}, z_{2}\right)$, i.e., functions which in a PCT $T$ of $D$ onto $D^{*}$ go over into functions $J_{D^{*}}{ }^{(\nu)}\left(z^{*}, \bar{z}^{*}\right), z^{*}=\left(z_{1}{ }^{*}, z_{2}{ }^{*}\right), \quad \nu=1,2, \cdots$, which at the corresponding points $z^{*}$ possess the same value as $J_{D}{ }^{(\nu)}$ at $z$. There are many different methods to determine invariants of $D$. There arises, however, the problem of determining domains $D$, for which the invariant $J_{D}^{(\nu)}$ is constant throughout $D$. The second problem is to find domains for which two different invariants, say $J_{D}{ }^{(\nu)}$ and $J_{D}{ }^{(\mu)}, \nu \neq \mu$, are linearly independent. In the present paper we investigate these problems for Reinhardt circular domains $R$. (For simplicity sake we assume that the center of $R$ is the origin $O$.)

Remark. A domain which admits the group $z_{k}{ }^{*}=z_{k} e^{i \varphi}, 0 \leqq \varphi \leqq 2 \pi$, $k=1,2$, of PCT's onto itself (automorphisms) is called a circular domain. See e.g., [B.-T., pp. 33-34]. A Reinhardt circular domain is a circular domain which admits the (two-parameter) group $z_{k}{ }^{*}=$ $z_{k} e^{i \varphi_{k}}, 0 \leqq \varphi_{k} \leqq 2 \pi$, of PCT's onto itself.

Since $J_{R}{ }^{(\nu)}$ is an analytic function of $z_{1}, z_{2}, \bar{z}_{1}, \bar{z}_{2}$, we have a series development of $1 / J_{R}{ }^{(\nu)}$ at $O$ in the form (9) of $\$ 1$, see p. 426 , and show

Received by the editors May 17, 1971.

AMS 1970 subject classifications. Primary $32-\mathrm{XX}$; Secondary $32 \mathrm{Hxx}, 32 \mathrm{H} 10$.

1This work was supported in part by AEC contract 326 Mod. III at Stanford University. 
that the coefficients $E_{n-p, p}^{(\nu)}$ are polynomials in $B_{00}^{-1}$ and $B_{r s}, r+s>0$, $r \geqq 0, s \geqq 0$. The $B_{r s}$ are geometric quantities of $R$, i.e., quantities which can be computed if the domain $R$ is given.

In $\$ 1$ the above polynomials $E_{n-p, p}^{(\nu)}\left(B_{r s}\right)$ are determined for the Reinhardt circular domains $R$. In this way we obtain a condition which permits us to decide whether or not $1 / J_{R}{ }^{(\nu)}$ is constant throughout the domain $R$. This method is again applied to determine a condition for $R$ in order that two invariants, say $J_{R}{ }^{(1)}$ and $J_{R}{ }^{(2)}$, are linearly independent.

In case if $1 / J_{R}{ }^{(v)}$ is not constant throughout the domain $R$, we investigate in $\$ 2$ the behavior of the hypersurfaces $1 / J_{R}{ }^{(\nu)}=$ constant. We consider three different cases. Since using the theory of the kernel function one can determine the hypersurfaces $1 / J_{D}{ }^{(v)}=$ constant for a large class of domains $D$, we obtain in $\$ 2$ a procedure to determine in $D$ the pseudo-conformal image $t=\boldsymbol{T}(O)$ of the center $O$ of $R$, provided that $D$ is a pseudo-conformal image of $R$. Once $t=\boldsymbol{T}(O), t=\left(t_{1}, t_{2}\right)$ is known, we determine the representative domain $\boldsymbol{R}(D, t)$, see [B.8, p. $187 \mathrm{ff}$ ]. If $D=\boldsymbol{T}(R)$, then $\boldsymbol{R}(D, t)$ can differ from a Reinhardt circular domain only by a linear (affine) PCT, see (72), p. 191 of [B.8]; in this case $\boldsymbol{R}(D, t)$ must be a circular domain.

Remark. Circular domains obtained by linear PCT's from Reinhardt circular domains will be investigated in a future paper.

In $\$ 3$ we discuss a special class of Reinhardt circular domains, namely,

$$
R_{p}=\left[\left|z_{1}\right|^{2 / p}+\left|z_{2}\right|^{2}<1\right], \quad p>0, p \neq 1,
$$

see [B.4] and [B.8, p. 197], and show that any two invariants of $R_{p}$ for each $p>0$ are linearly dependent. Therefore, a domain of this class has essentially one nontrivial (linearly independent) invariant. It would be most interesting to construct a domain explicitly in which there are two or more linearly independent invariants.

1. Conditions for the existence of nonconstant invariants in a Reinhardt circular domain. As mentioned in the introduction the use of the kernel function, among others, enables us to determine various invariants, i.e., real or complex functions which preserves their values in PCT's.

The functions

$$
1 / J^{(1)}(z, \bar{z})=K^{-1} \operatorname{det}\left(\frac{\partial^{2} \log K}{\partial z_{m}} \frac{\partial \bar{z}_{n}}{}\right),
$$




$$
\begin{aligned}
1 / J^{(\nu+1)}(z, \bar{z})=K^{-1} \operatorname{det}\left(\frac{\partial^{2}\left(1 / J^{(\nu)}\right)}{\partial z_{m} \partial \bar{z}_{n}}\right), \\
m, n=1,2 ; \nu=1,2, \cdots,
\end{aligned}
$$

see [B.6, p. $51 \mathrm{ff}],[B .7]$ and $\$ 4$, p. $182 \mathrm{ff}$. of [B.8], and the scalar curvature

$$
\tilde{J}(z, \bar{z})=\frac{1}{2} \sum_{m, n} T^{m \bar{n}} R_{m \bar{n}}, \quad m, n=1,2,
$$

are examples of invariants. Here $T^{m \bar{n}}$ are the contravariant components of the metric tensor $\left(T_{m \bar{n}}\right)$ and $R_{m \bar{n}}$ are the components of the Ricci curvature tensor, see [K.1] or [Y.B]. Investigating the question when a domain can be mapped pseudo-conformally onto a Reinhardt circular domain (or onto a general circular domain), it is useful to consider the level hypersurfaces

$$
1 / J^{(\nu)}(z, \bar{z})=c=\text { const, }
$$

since various properties of (3) are preserved in a PCT. The set defined by the relation (3) is as a rule three dimensional. However, it can happen that for some values of $c$, (3) degenerates to a twodimensional set or a point set. ${ }^{2}$

Remark. Here and in the following we formulate results for the invariants $1 / J^{(v)}$. Similar results are also valid for other invariants.

A simply connected domain $D$ of $C^{2}$ which possesses the property that its boundary, $\partial D$, can be written in the form

$$
s\left(\left|z_{1}\right|,\left|z_{2}\right|\right)=0
$$

where $s$ is a real continuous function of two real variables, ${ }^{3}$ is a Reinhardt circular domain with the center at the origin. It will be denoted by $R$.

REMARK. $R$ admits the group of automorphisms

$$
z_{k}^{*}=z_{k} \exp \left[i \varphi_{k}\right], \quad 0 \leqq \varphi_{k} \leqq 2 \pi, k=1,2 .
$$

Definition. A domain which can be obtained by a linear (affine) PCT

$$
z_{k}^{*}=A_{k 1} z_{1}+A_{k 2} z_{2}, \quad \operatorname{det}\left(A_{k v}\right) \neq 0, A_{k v}=\text { const, } k=1,2,
$$

from a Reinhardt circular domain will be denoted by $\hat{\boldsymbol{R}}$. The domains $\hat{R}$ obtained in this way are circular domains.

${ }^{2}$ As one can show it is impossible that the above set is one dimensional.

${ }^{3} s$ has to satisfy some additional conditions. See, e.g., [B.T., pp. 33-34] . 
It is at first of interest to decide for what circular domains, (1), (2) or other invariants are constant in the whole domain. If this is not the case, it is important to investigate the critical sets of (3) and indicate the conditions when these sets degenerate (for some values of $c$ ) to sets of lower dimension. The above properties of level hypersurfaces (3) are preserved under PCT's. Since in the case of an arbitrary (schlicht) bounded domain $D$, as a rule one can compute the hypersurfaces (3), we obtain in this way a necessary condition in order that $D$ is a pseudo-conformal image of a circular domain. As we shall show, using the same approach, one obtains also sufficient conditions in order that $D$ is pseudo-conformally equivalent to a domain $R$.

REMARK. A further class of domains which is of interest to investigate is the class of domains which can be obtained from Reinhardt circular domains by the PCT's

$$
z_{k}^{*}=\frac{A_{k 1} z_{1}+A_{k 2} z_{2}}{B_{k 1} z_{1}+B_{k 2} z_{2}}, \quad A_{k 1} B_{k 2}-A_{k 2} B_{k 1} \neq 0, k=1,2,
$$

where $A_{k n}$ and $B_{k n}$ are constants.

It is important that in the case of Reinhardt circular domains one obtains an answer to the above questions in terms of equation between certain geometric quantities of the domain $R$. The "moments"

$$
\begin{aligned}
\int_{D} z_{1}{ }^{m} z_{2}{ }^{n} \bar{z}_{1}{ }^{M} \bar{z}_{2}{ }^{N} d \omega, & \\
d \omega & =\text { volume element, } \quad m, n, M, N=0,1,2, \cdots,
\end{aligned}
$$

are examples of geometric quantities of $D$.

In the case of a Reinhardt circular domain, all the moments except

$$
\begin{aligned}
& B_{m p}^{-1}=\int_{R}\left|z_{1}\right|^{2 m}\left|z_{2}\right|^{2 n} d \omega, \\
& \qquad(m, p)=(0,0),(1,0),(0,1),(2,0), \cdots,
\end{aligned}
$$

vanish. The invariants $1 / J_{R}{ }^{(\nu)}(z, \bar{z}), \nu=1,2, \cdots$, of $R$ admit in the neighborhood of the center of $R$ (which we choose as the origin $O$, the development

$$
1 / J_{R}{ }^{(\nu)}(z, \bar{z})=\sum_{n=0}^{\infty} \sum_{p=0}^{n} E_{n-p, p}^{(\nu)}\left|z_{1}\right|^{2(n-p)}\left|z_{2}\right|^{2 p} .
$$

Here $E_{n-p, p}^{(\nu)}$ are polynomials in $B_{00}^{-1}$ and $B_{r s}, r+s>0, r \geqq 0, s \geqq 0$

THEOREM 1.1. The necessary and sufficient condition in order thai the invariant $1 / J_{R}{ }^{(v)}(z, \bar{z})$ is a constant is that all polynomials 


$$
E_{n-p, p}^{(\nu)} \equiv E_{n-p, p}^{(\nu)}\left(B_{r s}\right), \quad p=0,1, \cdots, n ; n=1,2, \cdots,
$$

vanish. (Infinitely many conditions.)

We proceed now to the determination of $E_{n-p, p}^{(\nu)}\left(B_{r s}\right), \nu=1,2$, for a Reinhardt circular domain $R$.

The kernel function $K_{R}$ of $R$ has the development

$$
K \equiv K_{R}=\sum B_{m p}\left|z_{1}\right|^{2 m}\left|z_{2}\right|^{2 p}, \quad B_{m p}^{-1}=\iiint_{R} \int\left|z_{1}\right|^{2 m}\left|z_{2}\right|^{2 p} d \omega
$$

at the origin. Here $d \omega$ is the volume element, and $\sum$ means the summation over

$$
(m, p)=(0,0),(1,0),(0,1),(2,0),(1,1),(0,2), \cdots .
$$

The invariants $1 / J_{R}{ }^{(\nu)}$ admit the developments

$$
\frac{1}{J_{R}^{(\nu)}}=\sum E_{m p}^{(\nu)}\left|z_{1}\right|^{2 m}\left|z_{2}\right|^{2 p}
$$

at the origin.

To obtain a recurrence formula for $1 / J^{(v+1)}$ in terms of the coefficients $E_{m p}^{(\nu)}$, we first compute $\operatorname{det}\left(\tilde{T}_{m \bar{n}}^{(\nu)}\right), \quad \tilde{T}_{m \bar{n}}^{(\nu)}=\partial^{2}\left(1 / J^{(\nu)}\right) / \partial z_{m} \partial \bar{z}_{n}$ :

$$
\begin{aligned}
& \tilde{T}_{11}^{(\nu)}=\sum_{n=1}^{\infty} \sum_{m+p=n} m^{2} E_{m p}^{(\nu)}\left|z_{1}\right|^{2(m-1)}\left|z_{2}\right|^{2 p} \\
& \tilde{T}_{12}^{(\nu)}=\bar{z}_{1} z_{2} \sum_{n=1}^{\infty} \sum_{m+p=n} m p E_{m p}^{(\nu)}\left|z_{1}\right|^{2(m-1)}\left|z_{2}\right|^{2(p-1)} \\
& \tilde{T}_{22}^{(\nu)}=\sum_{n=1}^{\infty} \sum_{m+p=n} p^{2} E_{m p}^{(\nu)}\left|z_{1}\right|^{2 m}\left|z_{2}\right|^{2(p-1)}
\end{aligned}
$$

Hence,

$$
\begin{aligned}
\operatorname{det}\left(\tilde{T}_{m \bar{n}}^{(\nu)}\right)=\sum_{q, t=1}^{\infty} \sum_{m+p=q} \sum_{r+s=n} & \\
& m s(m s-p r) \times E_{m p}^{(v)} E_{r s}^{(v)}\left|z_{1}\right|^{2(m+r-1)}\left|z_{2}\right|^{2(p+s-1)} .
\end{aligned}
$$

From this the coefficients of $\operatorname{det}\left(\tilde{T}_{m \bar{n}}^{(\nu)}\right)$ can be written down explicitly.

For example, if we write

$$
\operatorname{det}\left(\tilde{T}_{m \bar{n}}^{(\nu)}\right)=\sum_{k, l=0}^{\infty} P_{k l}^{(\nu)}\left|z_{1}\right|^{2 k}\left|z_{2}\right|^{2 l}
$$


then

$$
P_{k 0}^{(\nu)}=\sum_{m=1}^{k+1} m^{2} E_{m 0}^{(\nu)} \quad E_{k-m+1,1}^{(v)}
$$$$
P_{0 l}^{(v)}=\sum_{p=1}^{l+1} l^{2} E_{1, l-p+1}^{(\nu)} E_{0 p}^{(v)}
$$

$P_{k l}^{(\nu)}=\sum_{m+p=1}^{k+l+1} m(l+1-p)[m(l+1)-p(k+1)] E_{m p}^{(\nu)} E_{k-m+1, l-p+1}^{(\nu)}$.

Further, a formal computation shows that the coefficients of

$$
K^{-1}=\sum D_{m p}\left|z_{1}\right|^{2 m}\left|z_{2}\right|^{2 p}
$$

are given by

$$
D_{m p}=B_{00}^{-1} \sum_{k=0}^{m+p}(-1)^{k} \sum\left[\prod_{i=1}^{k}\left(B_{m_{i} p_{i}} B_{00}^{-1}\right)\right] .
$$

Here the second summation runs over all possible combinations of nonnegative integers for which

$$
\sum_{i=1}^{k} m_{i}=m, \quad \sum_{i=1}^{k} p_{i}=p, \quad m_{i}+p_{i} \geqq 1
$$

hold.

A formal computation yields for the coefficients $E_{m p}^{(1)}$ and $E_{m r}^{(2)}$ the expressions

$$
\sum_{1} B_{00}^{-q} \sum_{2} D_{k_{1} k_{2} \cdots k_{r}}^{l_{1} l_{2} \cdots l_{r}} \prod_{j=1}^{r} B_{k_{j} l_{j}}
$$

In the case of $E_{m p}^{(1)}, \sum_{1}$ means the summation is taken from $q=3$ to $q=3+m+p$, and in the case of $E_{m p}^{(2)}$ from $q=7$ to $q=9+$ $m+p . \sum_{2}$ means the summation over nonnegative integers $k_{j}, l$ such that

$$
\begin{aligned}
\sum_{j=1}^{r} k_{j}=m+1, & \sum_{j=1}^{r} l_{j}=p+1, \\
& 0<k_{j}+l_{j} \leqq m+p+1, j=1,2, \cdots, r, r=q-1,
\end{aligned}
$$

for $E_{m p}^{(1)}$, and 
TABLE $1^{*}$

Expression for $E_{m p}$ in terms of $B_{m p}$

\begin{tabular}{|c|c|c|c|c|c|c|c|c|c|c|c|c|c|c|c|c|c|c|}
\hline & \multicolumn{2}{|c|}{$E_{00}^{(1)}$} & \multicolumn{4}{|c|}{$E_{10}^{(1)}$} & \multicolumn{6}{|c|}{$E_{20}^{(1)}$} & \multicolumn{6}{|c|}{$E_{11}^{(1)}$} \\
\hline$q\left(\sum_{3}^{m+p+3}\right)$ & \multicolumn{2}{|c|}{3} & \multicolumn{2}{|c|}{3} & \multicolumn{2}{|c|}{4} & \multicolumn{2}{|c|}{3} & \multicolumn{2}{|c|}{4} & \multicolumn{2}{|c|}{5} & \multicolumn{2}{|c|}{3} & \multicolumn{2}{|c|}{4} & \multicolumn{2}{|l|}{5} \\
\hline \multirow{4}{*}{$\sum_{2} D_{k_{1} \cdots k_{4}-1}^{l_{1} \cdots l_{4-1}}$} & $D_{10}^{01}$ & 1 & $D_{11}^{(1)}$ & 1 & $D_{110}^{001}$ & -4 & $D_{12}^{(1)}$ & 1 & $D_{102}^{(1) 10}$ & -19 & $D_{1110}^{0001}$ & 10 & $D_{20}^{(02)}$ & 16 & $D_{002}^{110}$ & -16 & $D_{1}^{0}\left(\begin{array}{lll}000 & 1\end{array}\right.$ & 19 \\
\hline & & & $D_{02}^{10}$ & 4 & & & $D_{21}^{01}$ & 4 & $D_{111}^{001}$ & -4 & & & $D_{11}^{0.2}$ & 4 & $D_{110}^{(0) 12}$ & -16 & & \\
\hline & & & & & & & $D_{03}^{10}$ & 9 & & & & & $D_{02}^{11}$ & 4 & $D_{101}^{0111}$ & -9 & & \\
\hline & & & & & & & & & & & & & $D_{11}^{11}$ & -1 & & & & \\
\hline
\end{tabular}

Note. The terms of $E_{01}^{(1)}$ and $E_{02}^{(1)}$ can be found from the terms of $E_{10}^{(1)}$ and $E_{20}^{(1)}$, respectively, with $B_{m p}$ replaced by $B_{p m}$.

Example: $E_{00}^{(1)}=B_{10} B_{01} B_{00}^{-3}$,

$$
E_{10}^{(1)}=\left(B_{10} B_{11}+4 B_{01} B_{20}\right) B_{00}^{-3}-4 B_{10}^{2} B_{01} B_{00}^{-4}, E_{01}^{(1)}=\left(B_{01} B_{11}+4 B_{10} B_{02}\right) B_{00}^{-33}-4 B_{01}^{2} B_{10} B_{00}^{-4} \text {, etc. }
$$

*Tables of $E_{m p}^{(\nu)}\left(B_{r s}\right), \nu=1,2$, for a small number of $n$ have been published in a mimeographed report by $\mathrm{S}$. Bergman, "Interior distinguished points in the theory of functions of two complex variables" at Stanford University in collaboration with K. T. Hahn. It should be noted that some numerical errors occur in the previous tables and that the figures in the present tables are correct. 


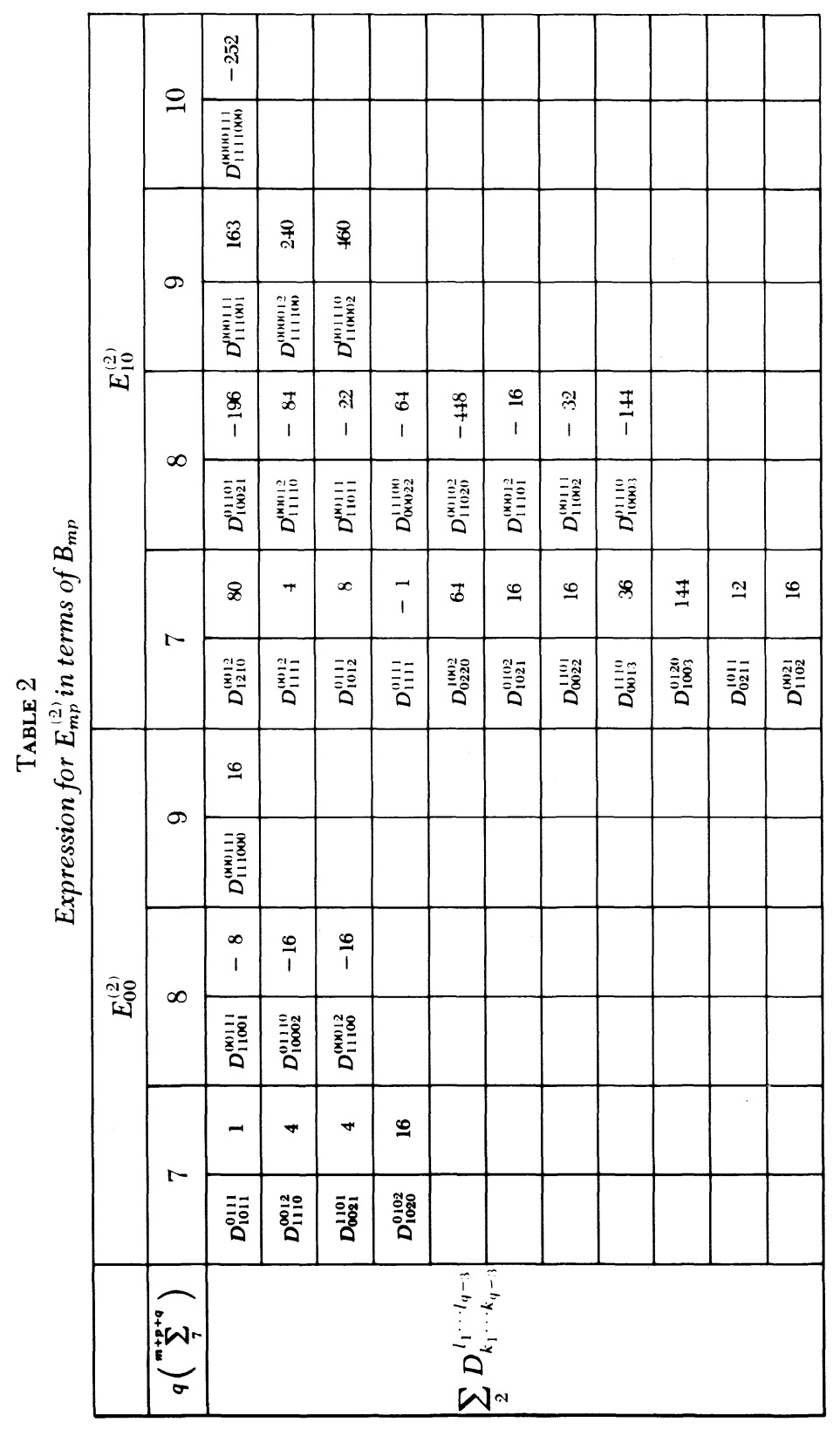

$++$

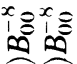

: :

i

صิ้

a

$\infty$

$1=1=$

उत्रें

에에

$\ddot{3} \infty$

요 8

हो I

Et

30.000

너?

4

a ++

Din

$\Xi \infty$

제

$+$

吾哈

$8 \infty$

छ

- $-\infty$

이용

4

in II II

है

से 


$$
\begin{aligned}
\sum_{j=1}^{r} k_{j}=m+3, & \sum_{j=1}^{r} l_{j}=p+3, \\
& 0<k_{j}+l_{j} \leqq m+p+2, j=1,2, \cdots, r, r=q-3,
\end{aligned}
$$

for $E_{m p}^{(2)}, D_{k_{1} k_{2} \cdots k_{r}}^{l_{1} l_{2} \cdots l_{r}}$ are integers for which one can obtain recurrence formulas. The values of the coefficients $D_{k_{1} k_{2} \cdots k_{r}}^{l_{1} l_{2} \cdots l_{r}}$ for $E_{m p}^{(1)}$ and $E_{m p}^{(2)}$ are found in Tables 1 and 2.

The method applied can also be used to determine conditions for $R$ in order that any two invariants $J_{R}{ }^{(v)}$ are linearly independent. As an example we derive a condition that $J_{R}{ }^{(1)}$ and $J_{R}{ }^{(2)}$ are independent. The necessary and sufficient condition in order that $1 / J_{R}{ }^{(\nu)}, \nu=1,2$, are linearly independent is that

$$
D^{(1,2)}=\frac{\partial\left(1 / J_{R}^{(1)}, 1 / J_{R}^{(2)}\right)}{\partial\left(\rho_{1}, \rho_{2}\right)} \not \equiv 0, \quad \rho_{k}=\left|z_{k}\right|^{2}, k=1,2 .
$$

Using (9) we obtain the series development for $D^{(1,2)}$ at $O$,

$$
D^{(1,2)}=\sum_{n=0}^{\infty} \sum_{p=0}^{n} H_{n-p, p}^{(1,2)} \rho_{1}{ }^{n-p} \rho_{2}{ }^{p},
$$

where $H_{n-p, p}^{(1,2)}$ are polynomials in $B_{00}^{-1}$ and $B_{r s}, r+s>0, s \geqq 0$, $r \geqq 0$. We obtain for $H_{00}^{(1,2)}$ again expression (20). Here $\sum_{1}$ means summation from $q=10$ to $13, \sum_{2}$ means summation over nonnegative integers $k_{j}, l_{j}$ such that

$$
\sum_{j=1}^{r} k_{j}=5, \quad \sum_{j=1}^{r} l_{j}=5, \quad 0<k_{j}+l_{j} \leqq 3, \quad j=1,2, \cdots, r, r=q-4 .
$$

The values of the coefficients $D_{k_{1} \cdots k_{y-4}}^{l_{1} \cdots l_{q_{-1}}}$ are found in Table 3.

THEOREM 1.2. Let $R$ be a Reinhardt circular domain. $H_{00}^{(1,2)} \neq 0$ is a sufficient condition in order that $J_{R}^{(1)}$ and $J_{R}^{(2)}$ are linearly independent.

2. The class of domains which are pseudo-conformally equivalent to a Reinhardt circular domain. By the PCT

$$
z_{k}^{*}=z_{k}-a_{k}, \quad k=1,2,
$$

where $a_{k}$ are conveniently chosen constants, a Reinhardt circular domain with the center at an arbitrary point $P=\left(a_{1}, a_{2}\right), \quad\left|a_{1}\right|^{2}+$ $\left|a_{2}\right|^{2}>0$, is transformed into a Reinhardt circular domain with a center at the origin $O$. Assume that $J_{R}{ }^{(v)}$ is not constant. The following three cases are possible: 
TABle 3

Expression for $H_{00}^{(1,2)}$ in terms of $B_{m p}$

\begin{tabular}{|c|c|c|c|c|c|c|c|c|}
\hline$q\left(\sum_{10}^{13}\right)$ & \multicolumn{2}{|c|}{10} & \multicolumn{2}{|c|}{11} & \multicolumn{2}{|c|}{12} & \multicolumn{2}{|c|}{13} \\
\hline & $D_{100112}^{011111}$ & 4 & $D_{1110011}^{0001112}$ & 32 & $D_{11000021}^{00111101}$ & 16 & $D_{111000002}^{000111110}$ & 128 \\
\hline & $D_{011111}^{100112}$ & -4 & $D_{0001112}^{1110011}$ & $-\quad 32$ & $D_{0011101}^{11000(1) 21}$ & -16 & $D_{00011110}^{111000002}$ & -128 \\
\hline & $D_{110102}^{001121}$ & 32 & $D_{1110110}^{0001112}$ & 72 & $D_{11100002}^{0001111}$ & 64 & & \\
\hline & $D_{001121}^{110102}$ & -32 & $D_{0001112}^{1110110}$ & -72 & $D_{00011111}^{11100002}$ & -64 & & \\
\hline & $D_{000113}^{111110}$ & 36 & $D_{1100021}^{001102}$ & 128 & $\begin{array}{r}D_{11000003}^{00111110} \\
\end{array}$ & 576 & & \\
\hline$\sum_{2} D_{k_{1} k_{2} \cdots k_{4-4}}^{l_{1} l_{2} \cdots l_{y-4}}$ & $D_{11110}^{000113}$ & -36 & $D_{0011102}^{1100021}$ & -128 & $D_{00111110}^{1100000(3)}$ & -576 & & \\
\hline & $D_{000221}^{111002}$ & 64 & $D_{0000221}^{1111001}$ & 272 & $D_{11111000}^{00000122}$ & 704 & & \\
\hline & $D_{111002}^{000221}$ & -64 & $D_{1111001}^{0000221}$ & -272 & $D_{00000122}^{1111000}$ & -704 & & \\
\hline & $D_{112100}^{000122}$ & 256 & $D_{1111010}^{0000113}$ & 288 & & & & \\
\hline & $D_{000122}^{112100}$ & -256 & $D_{0000113}^{111010}$ & -288 & & & & \\
\hline & $D_{100103}^{011120}$ & 288 & $D_{1110020}^{0001103}$ & 1152 & & & & \\
\hline & $D_{011120}^{100103}$ & -288 & $D_{0001103}^{1110020}$ & -1152 & & & & \\
\hline & $D_{110003}^{001220}$ & 576 & $D_{1000221}^{0111001}$ & 1280 & & & & \\
\hline & $D_{001220}^{110003}$ & -576 & $D_{0111001}^{1000221}$ & -1280 & & & & \\
\hline & $D_{002111}^{110111}$ & 16 & & & & & & \\
\hline & $D_{110111}^{002111}$ & -16 & & & & & & \\
\hline
\end{tabular}

Example: $H_{00}^{(1,2)}=\left(4 B_{10} B_{01}^{2} B_{11}^{2} B_{21}-4 B_{01} B_{10}^{2} B_{11}^{2} B_{12}+\cdots\right) B_{00}^{-10}+\left(32 B_{10}^{3} B_{01}^{2} B_{11} B_{12}-32 B_{01}^{3} B_{10}^{2} B_{11} B_{21}+\cdots\right)$

$\times B_{00}^{-11}+\cdots$. 


$$
\begin{aligned}
\mathrm{I}_{\nu}: & E_{10}^{(\nu)} \cdot E_{01}^{(\nu)}>0, \\
\mathrm{II}_{\nu}: & E_{10}^{(\nu)} \cdot E_{01}^{(\nu)}<0, \\
\mathrm{III}_{\nu}: & E_{10}^{(\nu)} \cdot E_{01}^{(\nu)}=0 .
\end{aligned}
$$

In the case of a Reinhardt circular domain of type $\mathrm{I}_{\nu}$, i.e., if there exists $J_{R}{ }^{\left({ }^{\prime}\right)}(z, \bar{z})$ satisfying (2a), the level hypersurface

$$
1 / J_{R}^{(\nu)}=E_{00}^{(\nu)}
$$

has a critical point (maximum or minimum point) at the origin. Therefore, if $D$ is a pseudo-conformal image of $R$, then the image of $O$ is a critical point (maximum or minimum) of one of the level hypersurfaces:

$$
1 / J_{D^{(\nu)}}(z, \bar{z})=c=\text { const }
$$

in $D$.

LEMMA 2.1. The level hypersurface (3) of a Reinhardt circular domain $R$ can degenerate to a point only at the origin $O$ (the center of $R$ ).

Proof. Let $P$ be the image of the Reinhardt circular domain in the $\rho_{1}, \rho_{2}$-space, $\rho_{k}=\left|z_{k}\right|^{2}$, see Fig. 2.1. To the level hypersurfaces (3) correspond in $P$ the lines

$$
1 / J_{P}^{(v)}\left(\rho_{1}, \rho_{2}\right)=c .
$$

Suppose that one level line of (5) for $c=c_{1}$ degenerates to a point, say $p_{0}$. If $p_{0}=\left(z_{1}{ }^{0}, z_{2}{ }^{0}\right)$ is such that $\left|z_{1}{ }^{0}\right|^{2}+\left|z_{2}{ }^{0}\right|^{2}>0$, then either $\left|z_{1}{ }^{0}\right|>0,\left|z_{2}{ }^{0}\right|>0$, or at least one of the above inequalities holds. If both inequalities hold, then to $p_{0}$ corresponds a torus in $R$. If $\left|z_{\nu}{ }^{0}\right|=0,\left|z_{3-\nu}^{0}\right|>0, \nu=1$ or 2 , then to $p_{0}$ corresponds in $R$ a circle which lies in $z_{\nu}=0$. Thus (3) degenerates to a point if and only if $p_{0}$ is the origin $\left(\rho_{1}, \rho_{2}\right)=(0,0)$ (the center of $R$ ).

Consequently it holds:

THEOREM 2.1a. Let $D$ be a domain in $C^{2}$ which possesses a kernel function. The necessary condition in order that the domain $D$ is a pseudo-conformal image of a Reinhardt circular domain $R_{\mathrm{I}_{\nu}}$ of the type $\mathrm{I}_{\nu}$ is that a level hypersurface (4) for a conveniently chosen value of $c$ or a segment of (4) degenerates to a point, say $t=\left(t_{1}, t_{2}\right)$. The point $t$ is the image of the center of $R_{\mathrm{I}_{\nu}}$.

THEOREM 2.1b. If $D$ is a pseudo-conformal image of $R_{\mathrm{I}_{\nu}}$, then there exists a point $t$, described in Theorem 2.1a, and the representative 
$\boldsymbol{R}(D, t)=\hat{R}$ is a domain which one can obtain from $R_{\mathrm{I}_{\nu}}$ by a linear transformation; $\hat{R}$ is a circular domain.

The proof of Theorem $2.1 \mathrm{~b}$ follows from the first theorem on p. 189 and the second theorem on p. 190 of [B.8]. Indeed, if $B^{*}$, see the second theorem on p. 190, is a Reinhardt circular domain $R_{\mathrm{I}_{\nu}}$ with the center at $t=t^{*}$, then $\boldsymbol{R}\left(R_{\mathrm{I}_{v}}, t\right)=R_{\mathrm{I}_{\nu}}$ and $\boldsymbol{R}(D, t)=\hat{R}$ can be obtained from $R_{\mathrm{I}_{\nu}}$ by a linear PCT (see Lemma 2.3).

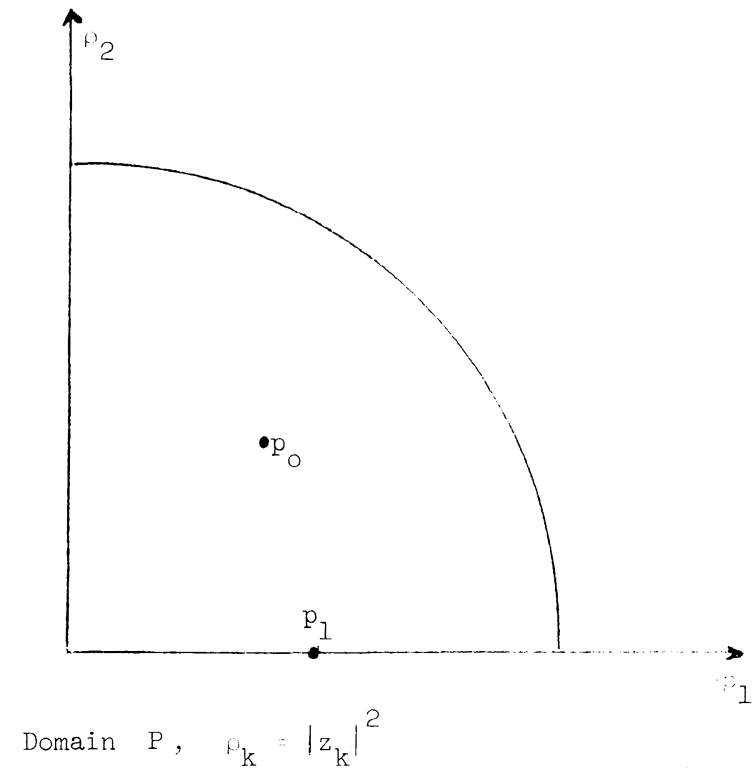

Figure 2.1

The case $\mathrm{II}_{v}$. Let $D$ be a pseudo-conformal image of a Reinhardt circular domain $R$ such that the coefficients of the development (9) of $\$ 1$ satisfy condition ( $2 b$ ). (If the development (9) of $\$ 1$ satisfies (2b), then $R$ will be denoted by $R_{\mathrm{II}_{\nu}}$.) In this case a three-dimensional hypersurface (4) (with $c=E_{00}^{(\nu)}$ ) passes through the center $O$ of $R_{\mathrm{II}_{\nu}}$. If in addition to the invariant $J^{(v)}$ a closed distinguished line, say $q^{1}$, lying on the distinguished hypersurface (3) is known, then we can determine in $D$ the location of the image of the center $O$ of $R_{\mathrm{II}_{\nu}}$.

The domain $R_{\mathrm{II}_{\nu}}$ admits the group of automorphisms (5) of $\$ 1$. Therefore, if the point, say $l, l \neq O$, lying on the hypersurface (3) is a distinguished point, then a closed curve $c^{1}$ lying on (3) is a distinguished line. Let $l=\left(l_{1}{ }^{0}, l_{2}{ }^{0}\right), \quad l_{k}{ }^{0} \neq 0, \quad k=1,2$, then the torus $T^{2}=$ 
$\left[z_{k}=l_{k}{ }^{0} \exp \left[i \varphi_{k}\right], k=1,2\right]$ is a distinguished surface. The intersection of (3) with $T^{2}$ is a closed curve $c^{1}$.

Lemma 2.2. Suppose that around each point of $c^{1}$ an invariant sphere of radius $\rho^{\prime \prime}$ is drawn. Then all these spheres have a point of intersection at the center of $R_{\mathrm{II}}$.

If we draw hyperspheres $H_{\rho}{ }^{4}$ with the centers along $c^{1}$ and of (noneuclidean) radius $\rho, \rho<\rho^{0}$, then there does not exist a point which belongs to all $\bar{H}_{\rho}^{4}$. For $\rho=\rho^{0}$ one point, the center of $R_{\mathrm{II}_{\nu}}$, will be the common point of intersection of all $\bar{H}_{\rho^{0}}^{4}$.

If $\rho>\rho^{0}$, then a four-dimensional domain will be the intersection of all $\bar{H}_{\rho}^{4}$.

Consequently it holds:

THEOREM 2.2. Let $D$ be a pseudo-conformal image of a Reinhardt circular domain $R_{\mathrm{II}_{\nu}}$. Then through the image of the origin passes a distinguished hypersurface $H^{3}=\left[1 / J_{R_{\mathrm{H}_{\nu}}}^{(\nu)}=E_{00}^{(\nu)}\right]$. Suppose that $h^{1}$ is a closed distinguished line lying on $\mathrm{H}^{3}$. If we draw around every point $p$ of $h^{1}$ an invariant hypersphere $H^{4}$ of radius $\rho$, then for a sufficiently small $\rho$ the intersection of all $\bar{H}^{4}$ will be empty. If $\rho$ increases continuously, then there exists a value of $\rho$, say $\rho=\rho^{0}$, so that all $\bar{H}^{4}$ intersect at one point, say $t$. For $\rho>\rho^{0}$, the intersection of all $\bar{H}^{4}$ is a four-dimensional set. Then $t=T(O)$, i.e., $t$ is the image of the center $O$ of $R_{\mathrm{II}_{\nu}}$.

Lemma 2.3. A circular domain $C$ with the center at the origin $O$ is mapped by a linear PCT (6) of $\$ 1$ onto a circular domain $C^{*}$ with the same center at $O$.

Proof. $C$ admits the group of automorphisms:

$$
\tilde{z}_{k}=z_{k} e^{i \varphi}, \quad 0 \leqq \varphi \leqq 2 \pi, k=1,2 .
$$

Let $C^{*}=\boldsymbol{T}(C)$, where $\boldsymbol{T}$ is the PCT $(6)$ of $\$ 1$. Let $\left(z_{1}{ }^{*}, z_{2}{ }^{*}\right)=\boldsymbol{T}\left(z_{1}, z_{2}\right)$ be a point in $C^{*}$. Then

$$
z_{k}^{*}=A_{k 1} z_{1}+A_{k 2} z_{2}=\left(A_{k 1} \tilde{z}_{1}+A_{k 2} \tilde{z}_{2}\right) e^{-i \varphi}=\tilde{z}_{k}^{*} e^{-i \varphi} .
$$

Therefore, $C^{*}$ admits the group

$$
\tilde{z}_{k}^{*}=z_{k}^{*} e^{i \varphi}, \quad 0 \leqq \varphi \leqq 2 \pi, k=1,2,
$$

of PCT onto itself.

LeMma 2.4. Suppose $D$ is a pseudo-conformal image of a Reinhardt circular domain with the image of the center at $t, t \in D$. Then $\mathbf{R}(D, t)$ is a circular domain with the center at $t$. 
Proof. By [B.8, p. $190 \mathrm{ff}$ ] , the domain $\boldsymbol{R}(D, t)$ can be obtained from a Reinhardt circular domain by a linear transformation. By Lemma $2.3 \boldsymbol{R}(D, t)$ must be a circular domain.

REMARK. Suppose that an invariant, say $\tilde{J}$, is known, such that .

$$
\frac{\partial\left(1 / J^{(v)}, \tilde{J}\right)}{\partial\left(\rho_{1}, \rho_{2}\right)} \not \equiv 0, \quad \rho_{k}=\left|z_{k}\right|^{2}
$$

then the intersection of $(5)$ with $\tilde{J}\left(\rho_{1}, \rho_{2}\right)=c=$ const for a conveniently chosen value of $c$ will be a point $\left(\rho_{1}{ }^{0}, \rho_{2}{ }^{0}\right), \rho_{k}{ }^{0} \neq 0$, yielding a torus $T^{2}$ in the $z_{1}, z_{2}$-space. The intersection of this torus with $H^{3}$ is the line $h^{1}$ considered in Theorem 2.2.

If one of the $\rho_{k}{ }^{0}, k=1,2$, vanishes, then the image of $\left(\boldsymbol{\rho}_{1}{ }^{0}, \boldsymbol{\rho}_{2}{ }^{0}\right)$ in the $z_{1}, z_{2}$-space is a curve which we can use as the curve $h^{1}$ in Theorem 2.2.

If the intersection

$$
\left[1 / J^{(\nu)}(z, \bar{z})=c_{1}\right] \cap\left[\tilde{J}(z, \bar{z})=c_{2}\right]
$$

is a torus $T^{2}$, then in Theorem 2.2 we can use $T^{2}$ instead of $h^{1}$.

In the case III $_{v}$ we consider the following two subcases: (a) $R$ possesses a distinguished set $s$ passing through $O$ and admits a group of automorphisms transforming an arbitrary point of $s$ into the origin $O$. Then an arbitrary point of $\boldsymbol{T}(s)$ can be used as the image of $O$ in $D=\boldsymbol{T}(R)$. (b) $R$ does not admit the group of automorphisms indicated in (a).

Assuming that a second invariant $\tilde{J}$ (linearly independent of $1 / J^{(\nu)}$ ) is known in both cases (a) and (b), we can proceed as in the case $\mathbf{I I}_{\nu}$. An example of a Reinhardt circular domain $R_{\mathrm{III}}$ possessing an interior distinguished surface $s=s^{2}$ is considered in [B.8, p. $197 \mathrm{ff}$.]

3. An example of a domain $R_{\mathrm{III}_{\nu}}$ In the cases $\mathrm{I}_{\nu}$ and $\mathrm{II}_{\nu}$ we obtained the series developments for the invariants around the center $O$. This permits us to determine the behavior of the invariant hypersurfaces $J^{(\nu)} \equiv$ const in the neighborhood of the center $O$.

It is of interest that for a class of domains, namely for

$$
R_{p}=\left[\left|z_{1}\right|^{2 / p}+\left|z_{2}\right|^{2}<1, p>0, p \neq 1\right],
$$

one can obtain for the kernel function (and consequently for most of the quantities connected with the invariant metric) expressions which are rational functions of $z_{1}, z_{2}$ and $\bar{z}_{1}, \bar{z}_{2}$ (see [B.4]). These results represent a useful illustration of our considerations. Further we show that any two invariants are linearly dependent for the Reinhardt circular domains $R_{p}$. 
In [B.4] and [B.11, p. 34], the kernel function $K_{p} \equiv K_{R_{p}}$ and the invariant $1 / J_{p}{ }^{(1)}$ are computed. We have for $R_{p}$

$$
\begin{aligned}
K_{R_{p}} & \equiv K_{p}=\left(1-\left|z_{2}\right|^{2}\right)^{p-2} D / \pi^{2} C^{3}, \\
1 / J_{R_{p}}^{(1)} & \equiv 1 / J_{p}^{(1)}=9 \pi^{2}\left(D^{4}-k^{2} C^{4}\right) / 2 D^{4}, \quad k^{2}=\left(p^{2}-1\right) / 3,
\end{aligned}
$$

where

(4) $C=\left(1-\left|z_{2}\right|^{2}\right)^{n}-\left|z_{1}\right|^{2}, D=(p+1)\left(1-\left|z_{2}\right|^{2}\right)^{n}+(p-1)\left|z_{1}\right|^{2}$.

A formal computation shows that the scalar curvature for $R_{p}$ is given by the rational function:

(5) $\tilde{J}_{p}=-1+\frac{4 k^{2} C^{4}\left(D^{4}-3 C D^{3}-6 k C^{2} D^{2}-k C^{3} D-3 k^{2} C^{4}\right)}{3\left(D^{2}-k C^{2}\right)\left(D^{2}+k C^{2}\right)^{3}}$.

It is interesting to observe that the scalar curvature $\tilde{J}_{p}$ assumes the maximum value, $\tilde{J}_{p}=-1$, on the boundary $\partial R_{p}$ and the minimum value, $\tilde{J}_{p}=-1-p(p-1)^{2} / 2(p+2)(2 p+1)^{2}$ on the plane $z_{1}=0$.

LEMMA 3.1. The domain functions

$$
\begin{aligned}
& X_{p}(z, \bar{z})=\left|z_{1}\right|^{2} /\left(1-\left|z_{2}\right|^{2}\right)^{p}, \\
& Y_{p}(z, \bar{z})=D(z, \bar{z}) / C(z, \bar{z}),
\end{aligned}
$$

where $C(z, \bar{z})$ and $D(z, \bar{z})$ are given in (4), define (pseudo-conformal) invariants of $R_{p}$, which are linearly dependent.

Proof. From (3),

$$
J_{p}^{(1)}=2 Y_{p}{ }^{4} / 9 \pi^{2}\left(Y_{p}{ }^{4}-k^{2}\right) \quad \text { or } \quad Y_{p}{ }^{4}=k^{2} J_{p}{ }^{(1)} /\left(J_{p}^{(1)}-2 / 9 \pi^{2}\right) .
$$

Since $J_{p}{ }^{(1)}(z, \bar{z})>2 / 9 \pi^{2}$ on $R_{p}, Y_{p}$ is a well-defined invariant of $R_{p}$. From the relation

$$
X_{p}=\left(Y_{p}-(p+1)\right) /\left(Y_{p}+(p-1)\right),
$$

and the inequality $p+1 \leqq Y_{p}<\infty$, it follows that $X_{p}$ is also a welldefined invariant of $R_{p}$. Clearly, $X_{p}$ and $Y_{p}$ are linearly dependent.

By (5) and (6b), $\tilde{J}_{p}$ can be expressed in terms of $Y_{p}$ by well-defined rational function. Therefore, we have

Corollary. Any two of the invariants $X_{p}, Y_{p}, 1 / J_{p}{ }^{(1)}$ and $\tilde{J}_{p}$ are linearly dependent in $R_{p}$.

Let $\pi: R_{p} \rightarrow Q_{p}{ }^{2} \subset \boldsymbol{R}^{2}$ be the projection map given by $\pi\left(z_{1}, z_{2}\right)=$ 
$\left(\boldsymbol{\rho}_{1}, \boldsymbol{\rho}_{2}\right), \boldsymbol{\rho}_{k}=\left|z_{k}\right|^{2}, k=1,2$, and $Y: Q_{p}{ }^{2} \rightarrow[p+1, \infty)$ by

$$
Y\left(\rho_{1}, \rho_{2}\right)=1+p \frac{\left(1-\rho_{2}\right)^{p}+\rho_{1}}{\left(1-\rho_{2}\right)^{p}-\rho_{1}},
$$

where $Q_{p}{ }^{2}=\left\{\left(\boldsymbol{\rho}_{1}, \boldsymbol{\rho}_{2}\right):\left(1-\boldsymbol{\rho}_{2}\right)^{n}>\boldsymbol{\rho}_{1}\right\} \subset \boldsymbol{R}^{2}$. Then under the composite map $Y \cdot \pi: R_{p} \rightarrow[p+1, \infty)$, the points on the plane $z_{1}=0$ correspond to the point $Y=p+1$ and the boundary points of $R_{p}$ correspond to $Y=\infty$.

LEMMA 3.2. For $p_{1} \neq p_{2}, p_{1}, p_{2}>0$, two domains $R_{p_{1}}$ and $R_{p_{2}}$ cannot be mapped onto each other by a PCT.

Proof. Suppose that there is a PCT, $w_{k}=w_{k}\left(z_{1}, z_{2}\right), k=1,2$, from $R_{p_{1}}$ onto $R_{p_{2}}$ and that $p_{1}>p_{2}$. Let $w_{k}(0,0)=w_{k}{ }^{0}$. Since $Y_{p_{1}}(0,0)=p_{1}+1$ and $Y_{p}$ is an invariant, $Y_{p_{2}}\left(w_{1}^{0}, w_{2}^{0}\right)=p_{1}+1$. But $Y_{p_{2}}\left(w_{1}^{0}, w_{2}^{0}\right) \leqq p_{2}+1$ which leads to $p_{1} \leqq p_{2}$.

LEMMA 3.3. Let $J_{p}(z, \bar{z})$ be any pseudo-conformal invariant of $R_{p}$. If $J_{p}$ depends linearly on $X_{p}, z \in R_{p}$, then all level hypersurfaces of $J_{n}$ are given by the equation:

$$
\left|z_{1}\right|^{2}=t\left(1-\left|z_{2}\right|^{2}\right)^{p}, \quad t \in[0,1) .
$$

Proof. If $J_{p}$ depends linearly on $X_{p}$ then it can be expressed by $X_{p}$ in the form of an (real) analytic function. Therefore, the totality of level hypersurfaces of one invariant coincide with the totality of level hypersurfaces of the other. Hence, all the level hypersurfaces of $J_{p}$ are given by the level hypersurfaces $X_{p}=t, t \in[0,1)$, which proves the lemma.

We note that any two distinct level hypersurfaces of a pseudoconformal invariant of $R_{p}$ which depends linearly on $X_{p}$ cannot be mapped onto each other by a holomorphic automorphism.

LeMma 3.4. The domain $R_{p}$ admits a group $G_{p}$ of holomorphic automorphisms:

$$
\begin{aligned}
& w_{1}=\left[\frac{\left(1-\left|t_{2}\right|^{2}\right)^{1 / 2}}{\left(1-\bar{t}_{2} z_{2}\right)}\right]^{p} z_{1} \exp \left[i \theta_{1}\right], \\
& \qquad \theta_{1}, \theta_{2} \in[0,2 \pi],\left(0, t_{2}\right) \in R_{p} . \\
& w_{2}=\frac{z_{2}-t_{2}}{1-\bar{t}_{2} z_{2}} \exp \left[i \theta_{2}\right],
\end{aligned}
$$

See [B.8, p. 197]. Conversely, all holomorphic automorphisms of $\boldsymbol{R}_{p}$ are given in the form (10). 
Proof. The first part of the lemma follows by the same method used in [B.8]. To prove the converse, we let $g$ be any holomorphic automorphism of $R_{p}$. By the previous remark, two points $z$ and $g z$ must lie on the same level hypersurface of $X_{p}$. Since $G_{p}$ acts transitively on each level hypersurface, there must be an element of $G_{p}$ which is identical to $g$. So $g \in G_{p}$ is of the form (10).

It is clear that $G_{p}$ contains the structural group $T_{p}$ of a Reinhardt domain as a subgroup. An element of $T_{p}$ is given by

$$
t\left(z_{1}, z_{2}\right)=\left(z_{1} \exp \left[i \theta_{1}\right], z_{2} \exp \left[i \theta_{2}\right]\right) .
$$

The orbit of $z^{0} \in R_{p}, \boldsymbol{O}_{T}\left(z^{0}\right)=\left\{\left(z_{1}^{0} \exp \left[i \theta_{1}\right], z_{2}{ }^{0} \exp \left[i \theta_{2}\right]\right): 0 \leqq\right.$ $\left.\theta_{1}, \theta_{2} \leqq 2 \pi\right\}$ with respect to $T_{p}$ forms a two-dimensional torus if $z_{1}{ }^{0} \neq 0, \quad z_{2}{ }^{0} \neq 0$. For $z^{0}=\left(0, z_{2}{ }^{0}\right)$ with $z_{2}{ }^{0} \neq 0$ or $\left(z_{1}{ }^{0}, 0\right)$ with $z_{1}{ }^{0} \neq 0, \boldsymbol{O}_{T}\left(z^{0}\right)$ is a circle. $\boldsymbol{O}_{T}\left(z^{0}\right)=z^{0}$ if and only if $z^{0}=(0,0)$. Since $G_{p}$ acts transitively on each level hypersurface of $X_{p}$ and any two distinct level hypersurfaces cannot be mapped onto each other by an element of $G_{p}$, the orbit $\boldsymbol{O}_{G}\left(z^{0}\right)$ of $z^{0} \in R_{p}$ with respect to $G_{p}$ coincides with the level hypersurface of $X_{p}$ passing through $z^{0}$. In particular, if $z^{0}$ lies on $z_{1}=0$ then $\boldsymbol{O}_{G}\left(z^{0}\right)$ is two dimensional and $\boldsymbol{O}_{G}\left(z^{0}\right)=\boldsymbol{O}_{G}(O)$. Thus, we have

LEMMA 3.5. On $R_{p}$ the orbit $\boldsymbol{O}_{G}\left(z^{0}\right)$ of a point $z^{0}=\left(z_{1}{ }^{0}, z_{2}{ }^{0}\right)$ with respect to $G_{p}$ forms a three-dimensional hypersurface if $z_{1}{ }^{0} \neq 0$, and a two-dimensional analytic surface if $z_{1}{ }^{0}=0$. Furthermore, such orbits coincide with level hypersurfaces of $X_{p}$.

THEOREM 3.1. On $R_{p}$ any two pseudo-conformal invariants $J_{p}{ }^{(1)}$ and $J_{p}{ }^{(2)}$ are linearly dependent.

Proof. First we note that an invariant of $R_{p}$ cannot take a constant value on a four-dimensional set of $R_{p}$, unless it reduces to a constant. On the other hand, an invariant must take a constant value on each orbit and hence its level hypersurfaces are of dimension two or three. It is therefore clear that the totality of the level hypersurfaces of the invariant $J_{p}{ }^{(1)}$ includes all the orbits of $R_{p}$ with respect to $G_{p}$. Conversely, for any level hypersurface of $J_{p}{ }^{(1)}$ there exists exactly one orbit to which the level hypersurface coincides. In fact, let $z^{0}$ be a point on the level hypersurface, i.e., $J_{p}^{(1)}(z, \bar{z})=c=J_{p}{ }^{(1)}\left(z^{0}, \bar{z}^{0}\right)$. Since $R_{p}=\bigcup_{z \in R_{p}} \boldsymbol{O}_{G}(z)$, there exists exactly one orbit which passes through $z^{0}$. Since $\boldsymbol{O}_{G}\left(z^{0}\right)$ is homogeneous, $J_{p}^{(1)}(z, \bar{z})=c$ for all $z \in \boldsymbol{O}_{G}\left(z^{0}\right)$. Suppose that there exists a point $\tilde{z}$ on the level hypersurface such that $\tilde{z} \notin \boldsymbol{O}_{G}\left(\boldsymbol{z}^{0}\right)$. Then again there exists another orbit $\boldsymbol{O}_{G}(\boldsymbol{z})$ on which $J_{p}^{(1)}(z, \bar{z})=J_{p}^{(1)}(\tilde{z}, \widetilde{\tilde{z}})=c$. Since two orbits are either identical or disjoint, the level hypersurface $J_{p}{ }^{(1)}(z, \bar{z})=c$ consists of 
two disjoint orbits, which is impossible. Thus, by Lemma 3.5 the totality of level hypersurfaces of $J_{p}{ }^{(1)}$ and $X_{p}$ coincide. The same holds for $J_{p}{ }^{(2)}$ and $X_{p}$. This completes the proof.

\section{BIBLIOGRAPHY}

[B.1] S. Bergman, Ueber Hermitesche unendliche Formen, die zu einem Bereich gehören, nebst Anwendungen auf Fragen der Abbildung durch Funktionen zweier komplexen Veränderlichen, Berichte Berliner math. Gesellschaft 26 (1927), 178-184; Math. Z. 29 (1929), 640-677.

[B.2] - Ueber die Existenz von Repräsentantenbereichen, Math. Ann. 102 (1929), 430-446.

[B.3] — Ueber die Kernfunktion eines Bereiches und ihr Verhalten am Rande, J. Reine Angew. Math. 169 (1933), 1-42; ibid. 172 (1934), 89-128.

[B.4] _ Zur Theorie von pseudokonformen Abbildungen, Mat. Sb. 1 (43) (1936), 79-96.

[B.5] - The behavior of the kernel function at boundary point of the second order, Amer. J. Math. 65 (1943), 679-700. MR 5, 95.

[B.6] - Sur les fonctions orthogonales de plusieurs variables complexes avec les applications à la théorie des fonctions analytiques, Interscience, New York, 1941; Mémor. Sci. Math., no. 106, Gauthier-Villars, Paris, 1947. MR 2, 359; MR 11, 344. (English translation to appear.)

[B.7] —, Sur la fonction-noyau d'un domaine et ses applications dans la théorie des transformations pseudo-conformes, Mémor. Sci. Math., no. 108 Gauthier-Villars, Paris, 1948. MR 11, 344. (English translation to appear.)

[B.8] —, The kernel function and conformal mapping, 2nd ed., Math Surveys, no. 5, Amer. Math. Soc., Providence, R. I., 1970.

[B.9] —, Distinguished sets of invariants in the theory of pseudo-conforma transformations, J. Analyse Math. 13 (1964), 317-353. MR 31 \#5987.

[B.10] — On boundary behavior of some domain functionals in the theor of functions of two complex variables, Bull. Soc. Sci. Lettres Kódź 14 (1966) $1-15$.

[B.11] - Some properties of pseudo-conformal images of circular domain: in the theory of two complex variables, Proc. Conference Complex Analysi: (Minneapolis, Minn., 1964), Springer, Berlin, 1965, pp. 30-40. MR 32 \#2612

[B.12] - Interior distinguished points in the theory of functions of twe complex variables, J. Analyse Math. 18 (1967), 7-21. MR 35 \#4468.

[B.13] —, On pseudo-conformal images of circular domains, J. Analys Math. 22 (1969), 269-284. MR 40 \#2901.

[B.T.] H. Behnke and P. Thullen, Theorie der Funktionen mehrerer complexe Veränderlichen, Zweite, erweiterte Auflage, Ergebnisse der Mathematik unı ihrer Grenzgebiete, Band 51, Springer-Verlag, Berlin and New York, 1970.

[F.1] B. Fuks, Ueber geodätische Mannigfaltigkeiten einer bei pseudo konformen Abbildungen invarianten Riemannschen Geometrie, Mat. Sb. 2 (4 (1937), 567-594.

[F.2] - Special chapters in the theory of analytic functions of severc complex variables, Fizmatgiz, Moscow, 1963; English transl., Transl. Matł Monographs, vol. 14, Amer. Math. Soc., Providence, R. I., 1965. MR 30 \#497c MR 32 \#5915.

[H.1] K. T. Hahn, Minimum problems of Plateau type in the Bergman metri space, Pacific J. Math. 14 (1964), 943-955. MR 29 \#6402. 
[K.1] S. Kobayashi, Geometry of bounded domains, Trans. Amer. Math. Soc. 92 (1959), 267-290. MR 22 \#3017.

[W.1] H. Welke, Über die analytischen Abbildungen von Kreiskörpern und Hartogsschen Bereichen, Math. Ann. 103 (1930), 437-449.

[Y.B.1] K. Yano and S. Bochner, Curvature and Betti numbers, Ann. of Math. Studies, no. 32, Princeton Univ. Press, Princeton, N. J., 1953. MR 15, 989.

Stanford University, Stanford, California 94305

Pennsylvania State University, University Park, Pennsylvania 16802 


\section{.}

